\title{
Building a human behaviour community
}

\author{
Individual and collective human behaviour is studied by numerous fields, spanning the social and \\ natural sciences and beyond. Genuine progress in understanding human behaviour can only be achieved \\ through a multidisciplinary community effort. Nature Human Behaviour aims to foster that effort.
}

The study of human behaviour is key to improving the human condition and addressing pressing societal challenges discrimination, poverty, inequality, conflict, unsustainable development, disease.

Progress in achieving any of the 17 Sustainable Development Goals of the 2030 Agenda for Sustainable Development partly hinges on making progress in understanding, predicting, and changing human behaviour. Nonetheless, research in human behaviour has had no dedicated home. There are excellent discipline-specific journals serving each of the many fields that study human behaviour, but no forum for the publication of the most timely and broadly relevant research and opinion from across disciplines.

Nature Human Behaviour fills this gap, offering an outlet for cutting-edge research from any of the social and natural sciences that has a bearing on understanding human behaviour - its causes and consequences, its biological bases, the contexts that shape it, its evolutionary and developmental trajectories, its collective dynamics, the ways it can break down as well as the ways it can be changed. We welcome submissions from a very broad range of disciplines including, but not limited to, psychology, economics, political science, sociology, anthropology, geography, epidemiology, behavioural ecology, evolutionary biology, neuroscience, psychiatry, genetics, physics and computer science - that represent a major leap forward in describing, explaining, predicting or changing human behaviour.

We want to publish the research that matters the most to each field but that will also be of interest and influential for others working on human behaviour from different angles. We particularly welcome interdisciplinary research that combines theory or methods from two or more disciplines, as well as research that engages with urgent societal issues.

Scientific advances take different forms - some of the research we will be publishing will be characterized by high conceptual novelty; other by innovative methods; still other by its direct relevance in addressing a pressing societal challenge. Science advances by both discovery and (dis)confirmation, though, and for this reason we will also be publishing high-value replication studies, as well as studies that, due to their scale and rigour, supersede earlier research and significantly strengthen our confidence in a scientific discovery or convincingly dispel it.

Some of the disciplines that study human behaviour have long and rich individual traditions; others are newer arrivals, spun from recent technological advances or societal needs. The methods used in studying human behaviour are as varied as the disciplines that study it but none takes precedence over the other in this journal. We are open to research using any methods - be they experimental or observational; based on primary or secondary data; formal or computational; qualitative, mixed, or case studies. What we are looking for is 'best in class' research that uses methods appropriate for the question asked.

Our first issue provides a flavour of what we intend the journal to be - the go-to place for leading-edge research, opinion, debate, and news on human behaviour science and its societal impacts. More than 15 distinct disciplines are represented in the research and magazine sections of this issue, including psychology, geography, epidemiology, anthropology, political science, social policy, education, evolution, neuroscience, and genetics. The research section features articles using a variety of methods to tackle diverse questions, such as: what drives changes in gender inequality (article no. 0003)? How does the prevalence of social phenomena, such as crime, innovation and education, change with population size (article no. 0012)? Can fear be reduced unconsciously (article no. 0006)? How do we infer personality traits from people's faces (article no. 0001)? What are the causes and genetic consequences of assortative mating - people choosing partners who are similar to themselves (article no. 0016)?

The magazine section includes thought-provoking perspectives, incisive commentary, highlights from the literature and more, focusing on issues of societal relevance and significance. For instance, Duncan Watts highlights in a Perspective (article no. 0015) the negative consequences of disciplinary silos and advocates for social science to place more emphasis on solving practical problems that are of direct interest to decision makers and the public. Gary Slutkin argues that violence of any form (from street violence to war) can be effectively reduced if it is recognized as a contagious epidemic and treated with the methods of epidemic control (article no. 0025). James Heckman and Jorge Luís García discuss (article no. 0019) the social policy implications of research reported by Caspi et al. (article no. 0005), which shows that a small segment of the population accounts for a disproportionate share of costly service use in a society's health-care and social welfare systems, and that this group can be predicted with high accuracy from as early as age 3 years. Elke Weber explains in a Comment (article no. 0013) the cognitive biases that stand in the way of making decisions for a sustainable future and how they can be overcome. Featured in other pieces are authoritative, insightful perspectives on policymaker behaviour, education policy, corruption, voter emotions and electoral decision-making, and the impact of technology on behaviour and the brain.

The journal is committed to publishing robust, rigorous science (see also companion Editorial) and incisive, provocative opinion. We will not shy away from covering controversial topics and will strive for pluralism of voices, including engaging stakeholders outside academia.

Ultimately, we aim to create a community space for all researchers working on human behaviour and those interested in advances in human behaviour research to interact, engage, and stay up to date with the most significant developments in the field. By fostering a diverse multidisciplinary community we hope to enable human behaviour research fulfil its mission of improving the human condition. 